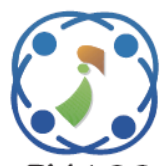

\title{
Adaptive Duty-Cycle Scheme Based on a New Prediction Mechanism for Energy Optimization over IEEE 802.15.4 Wireless Network
}

\author{
Imad Iala $^{1 *} \quad$ Imane Dbibih $^{1} \quad$ Ouadoudi Zytoune $^{2}$ \\ ${ }^{1}$ LRIT Associated Unit to the CNRST-URAC N29, Faculty of Sciences, \\ University Mohammed V, B.P.1014 RP, Rabat IT center, Morocco \\ ${ }^{2}$ ENSA, University Ibn tofail Kenitra, Morocco \\ * Corresponding author's Email: imad.iala01@gmail.com
}

\begin{abstract}
One of the toughest challenges encountered in Wireless Sensor Networks (WSNs) is the limited power resources, which directly affects the network services quality and the sensors lifetime. Therefore, the proposed protocols must prolong the network lifetime as long as possible by minimizing the major sources of energy waste duo to the idle listening (no sent or received packets by the sensor). In this paper we propose a new communication protocol based on IEEE 802.15.4 standard called Adaptive Duty-cycle (AD- MAC) that aims at reducing the energy dissipation where the transceiver is waiting potential packets. AD-MAC protocol mainly intends to reduce the power consumed by sensors in Beacon-Enabled mode via a dynamic duty-cycling technique. AD-MAC protocol optimizes the sleep interval between consecutive wake-ups without negatively influencing network performance. This solution uses a new predicting mechanism to estimate the moment when the sensors wake up or sleep according to previous traffic load statistics. Simulation results via NS-2 simulator show that AD-MAC protocol outperforms IEEE 802.15.4 standard in terms of minimizing energy consumption, and Packet Delivery Ratio (PDR).
\end{abstract}

Keywords: WSN, Mac protocol, IEEE 802.15.4 standard, Energy consumption, Prediction mechanism.

\section{Introduction}

The general purpose of WSNs is to collect a set of environmental parameters surrounding the nodes, such as atmospheric pressure or temperature in order to transfer it to the sink node. WSNs are considered as the most used networks in several types of applications such as military, healthcare, microsurgery and environmental monitoring [1 - 3]. This typical network must responds mainly to many constraints; namely Auto-configuration, cost of devices, number of deployed nodes and energy consumption. Despite this latter that dependents on many parameters (e.g. sensing, processing and communication), the radio interface unit is generally the most energy consumer component. However, energy conservation is considered as the most important challenge in WSNs designing because it has a direct impact on the autonomy and the network lifetime. To address this issue, it is important to implement new solutions to reduce energy consumption.

Duty-cycling approaches are the most used techniques for minimizing sensor energy consumption and extending the network lifetime. In these approaches, network nodes switch the radio interface between two states; sleep and active states. In the sleep state, the sensor nodes turn off the transceiver in order to conserve more energy. And, in the active state, nodes activate the transceiver and became ready to receive or send data.

In this paper, a new predictive technique is developed to analyze the energy consumption, latency and PDR of network nodes and to study and evaluate the impact of various network parameters such as the nodes number and the data rate. The main process of $\mathrm{AD}-\mathrm{MAC}$ protocol is to estimate the moments when the data reception may occur. According to the result of this process, node decides to become in active or sleep state during the 
Contention Free Period (CFP) time slots of the super-frame. AD-MAC protocol does not require any central coordination, central computation, or global time synchronization. Thus, it is scalable to new entrant nodes to the network and variable packet lengths. And it is also robust to system errors, such as inaccurate schedule.

The remainder of this paper is as follow: Section 2 gives an overview of several related works. Section 3 describes the main process of AD-MAC protocol and its details. Section 4 summarizes the results given by NS2 simulator and provides results interpretations. Finally, we address some prospects and we conclude the paper in Section 5.

\section{Related works}

In literature there are lots of studies that addressed the WSN problems and proposing the solutions that can be applied at the MAC layer. The proposed MAC protocols aim at reducing the energy dissipation due to idle listening problem, and they are based on the same duty-cycle principle in order to extend the network lifetime by optimizing the transceiver energy consumption.

In [4], the authors proved that the listening process consumes $50 \%$ to $100 \%$ of the energy. The recent works such as [5] adopted a synchronized Sleep/Wake up cycle to allow nodes to save energy. Several research works have been proposed in the context of maximizing the devices lifetime and which are based on the IEEE 802.15.4. In [6], the authors proposed GTS (Guaranteed Time Slot) Size Adaptation Algorithm (GSAA) that defines a new GTS allocation scheme that will set the GTS size for it to be adapted according to the data size of the devices. The authors of [7] presented a cross-layer solution to the problem of PAN coordinator election; this solution is a process topology formation of the network operating at the MAC layer of the IEEE 802.15.4 using a topology reconfiguration algorithm defined at the network layer. In [8], the authors proposed an approach that improves the Adaptive Collision Resolution (ACR); this approach adjusts Backoff Periods (BP) to avoid timing and direction of additional Clear Channel Assessment (CCA) based on the estimated remaining time that the channel becomes inactive. The authors of [9] consider an adaptive CSMA/TDMA hybrid channel access method to resolve some weaknesses in the slotted CSMA/CA method in order to minimize the power consumption. In [10], the authors proposed an efficient slot reservation MAC protocol to reduce energy consumption and improve the efficiency in transmitting collected data in wireless sensor networks.

The [11] have proposed a new algorithm called Duty Cycle Learning Algorithm (DCLA), this latter adjusts the duty cycle during run time in order to save energy consumption while balancing probability of successful data delivery and delay constraints of the application. The authors of [12] propose a new MAC protocol of QoS, "PRIority in Node" (PRIN), using static priority in the source and the intermediate node and priority among the node which is one hop from the sink node to achieve QoS in WSNs. The [13] have proposed a new multi-site MAC protocol called the Opportunistic MAC WakeUp (OPWUM), which uses timer-based contention. This protocol allows the opportunistic selection of the best receiver among its neighboring nodes according to a given metric (for example, the remaining energy) and exploits the emerging alarm receivers to reduce the nodes energy consumption. In [14], the authors have modeled and simulated a real Wakeup Radio (WuR) hardware platform developed by the authors themselves on the OMNET ++ simulator. Energy efficiency, latency and PDR of the WuR platform are compared with the performance of four of the MAC protocols widely used for WSN under three real network deployments. The authors of [15] used the Markov chain to model the waiting packets in the node's transmit/receive buffer and calculate the probability of buffer overflow. They also formulated the optimization problem (OP) that minimizes the power consumption of the node, the probability of buffer overflow and data rate. In addition, they have presented the OP-Based Energy Management System (OBEMS) which uses the OP solution. Their results show that the proposed scheme reduces energy consumption while maintaining a higher PDR. In [16] the authors aim to optimize the utilization of energy at each network by proposing a WSN Routing Protocol. The proposed protocol is named as An Efficient Routing Protocol Adopting Enhanced Cluster Formation Technique Accompanied by Fuzzy Logic for Maximizing Lifetime of WSN. This protocol is based on the PSO algorithm to assign some of the nodes that have been omitted to current clusters to eliminate the rate of formation of individual nodes. And for the $\mathrm{CH}$ selection, the authors have been based on parameters such as residual energy, distance to neighbor, density, maximum distance and angle.

The authors of [17] proposed a Cross Layer Interactive Framework (CLIF) to balance the importance of critical performance metrics such as data delivery and energy efficiency in order to cater 
up the demands of real-time applications under dynamic environment. The CLIF protocol assigns a high degree of significance (high priority) to critical messages to valorize them in the processing. An optimized QoS routing protocol (OQoS-MRP) for wireless sensor networks is proposed by the authors of [18]. This protocol is designed to calculate an optimal multi-hop path between the transmitter and the receiver. Based on various QoS parameters such as reliability, energy, delay, distance to sink and hop count, OQoS-MRP protocol chooses the best path for transferring the data to the sink.

\section{AD-MAC process}

In this article, we are interested by the deterministic MAC protocols where we aim to reduce the energy consumption and to control the medium access according to the previous statistics of traffic load. AD-MAC protocol is based on IEEE 802.15.4 [15, 16] standard. Before presenting our protocol in the following a brief overview of IEEE 802.15.4 operating.

The IEEE 802.15.4 is the widely used protocol for Low Rate Wireless Personal Area Networks (LR-WPANs) at the present. Thanks to its low energy consumption and low duty-cycle, the IEEE 802.15.4 standard is considered as the reference networking standard for WSNs. For example, it is used by ZigBee technology to process data in the physical and MAC sub-layers. The IEEE 802.15.4 standard allows network nodes to enable their transmitter to occupy the transmission channel for a few milliseconds, and after receiving a response or acknowledgment, they fall asleep for a long period before next transmission. Therefore, they can conserve more energy.

In The IEEE 802.15.4 standard we have two types of network nodes: FFD (Full Function Device) implementing the entire standard specification, while RFD (Reduced Function Device) is a low capacity device. The latter entities can only be terminal nodes of the network.

IEEE 802.15.4 standard offers two access modes to the medium, a non-beacon enabled mode and a beacon enabled one. In the first mode, there is no emission of beacon frame, thus there is no synchronization between the different network nodes. Nodes wishing to transmit data have to use the non Slotted CSMA/CA algorithm (i.e. once the medium appears free the node can transmit its data).In the second mode, the network coordinator node periodically broadcast beacon frames. Each one contains information about network

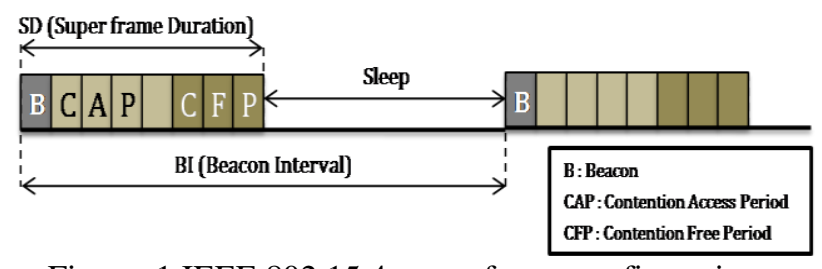

Figure. 1 IEEE 802.15.4 super-frame configuration

configuration, and some data allowing network nodes to know the times when they should wake up to synchronize themselves.

The time interval between two beacons frames is called super-frame. It includes two periods, active and inactive parts. The first period is divided into several time slots of equal durations; the beacon always occupies the slot 0 and allows the synchronization of all nodes within the same coordinator range. Typically, the network nodes just wake up before slot 0 and start listening. After receiving a beacon frame from the coordinator node, the nodes become aware of the current super-frame configuration. If they have no data to transmit, or to receive, they can doze until the next beacon; otherwise they fall asleep as soon as the transaction (transmission and/or reception) is finished. Fig.1 shows the IEEE 802.15.4 super-frame configuration.

The IEEE 802.15.4 standard offers two access modes within the super-frame (see Fig.1), the first one concerns the Contention Access Period (CAP): Here, the access is done according to the slotted CSMA/CA technique. The second concerns the Contention Free Period (CFP) "Optional", in which the medium access is managed by the network coordinator node (i.e. deterministic access) that can allocate one or more Guaranteed Time Slots (GTS) to network nodes.

Our solution is based on the IEEE 802.15.4 standard employing the Beacon-Enabled mode wakeup scheme as well as Clear Channel Assessment (CCA) and slotted CSMA/CA mechanism to access the channel. The network coordinator node periodically broadcasts beacons frames, and we assume that it has no energy constraint. Then, we should not be worry about its energy consumption.

Each network node applies the same operational cycle, which consists of two periods: wake up and sleep period. The sleep period in which a node conserves its energy consumption by turning off the radio interface. And the wake-up period in which a node is ready for receiving or transmitting data when its radio interface is activated.

The wake-up period is divided into several time slots: each time slot can be either in active or 


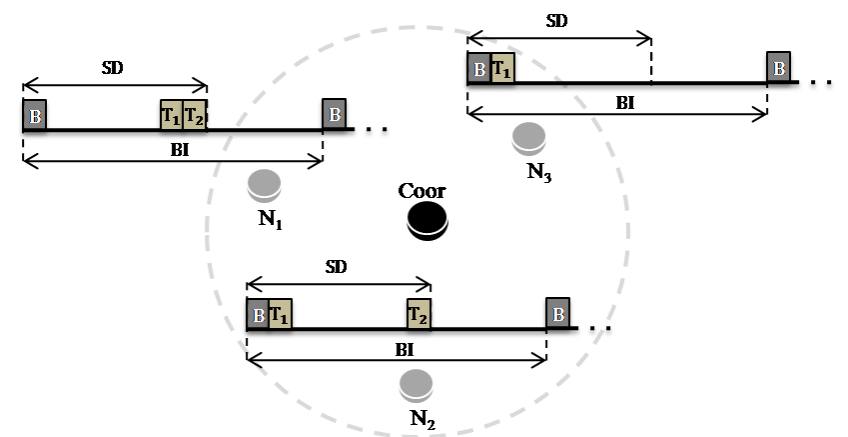

Figure. 2 AD-MAC super-frame configuration

inactive state according to a distributed processing based on a prediction mechanism. This prediction mechanism allows network nodes to estimate the moment when a data reception may occur and consequently, nodes decide to become in active or inactive state during a time slot, which explains why each network node will have its own duty-cycling scheme (see Fig.2). I-e, the prediction mechanism will adapt the duty-cycling scheme to node traffic load.

In Fig.2, the basic operation of our solution is shown. Each sensor node wakes up according to its own schedule to check if there are any incoming data intended for it.

The node that needs to transmit data must wait for the active period (SD) to turn on its radio interface and uses the slotted CSMA/CA technique to access the channel.

In order to model the sensor nodes radio interface with a dynamic duty-cycling scheme, we consider a network composed of a certain number of nodes that operate according to a specific SD configuration. We also consider that the active part of the SD is composed of a number of Times Slot (TS) noted NTS. A node receives the data packets at times $t_{k, 1}, t_{k, 2}, t_{k, 3}, \ldots, t_{k, n i} \in R$ where $k \epsilon[1, N T S]$, and $n_{i}$ is the number of packets received at time slot $i$. These packets arrive at each node independently.

At the beginning, all TS of SD are kept in active state. Then, Each data receiving instant in a TS have awaking probability noted: $W=\left[w_{1,1}, \ldots, w_{1, n l}, \ldots\right.$, $\left.w_{2,1}, \ldots, w_{2, n 2}, \ldots, w_{N T S, 1}, \ldots, w_{N T S, n N T S}\right] \epsilon R$, which will depend on the number of received packets and which shall computed according to Eq. (1).

$$
W_{i, j}=\frac{\sum_{i=1}^{N T S} \text { Number of received packets }}{\sum_{i=1}^{N T S} \sum_{j=1}^{n i} \text { Number of received packets }}
$$

Where $i \epsilon[1, N T S]$. In other words, all received packets instants in the same TS will have the same awakening probability. Then, our system can be described by the following equation:

$$
Y=\sum_{i=1}^{N T S} P_{i} \sum_{j=1}^{n i} W_{i, j} t_{i, j}+\sigma n
$$

We put that $n \triangleq \sum_{i=1}^{N T S} n_{i}$, that corresponds to the all received packets during the previous super-frame. With $P \in R^{n * n}$ is a diagonal covariance matrix between TS with inputs $P_{i}$ and multiplicity $n_{i}$ for $i=1, \ldots, N T S$, and $T=\left[t_{1}{ }^{t} ; \ldots ; t_{N T S}{ }^{t}\right] \epsilon R^{n}$, where $t_{k} \epsilon$ $R^{n i}$ for $i=1, \ldots, N T S$, and $t_{k}$ is a vector with $j^{\text {th }}$ input $t_{k, j}$.

$$
\begin{aligned}
& \left(\begin{array}{ccc}
P_{1} & \cdots & 0 \\
\vdots & \ddots & \vdots \\
0 & \cdots & P_{1}
\end{array}\right) \quad \mathbf{0} \quad \mathbf{0} \\
& \begin{array}{llll}
P= & \text { 0 } & \ddots & \text { 0 }
\end{array} \\
& \mathbf{0} \quad \mathbf{0}\left(\begin{array}{ccc}
P_{n} & \cdots & 0 \\
\vdots & \ddots & \vdots \\
0 & \cdots & P_{n}
\end{array}\right)
\end{aligned}
$$

We obtain the vector $Y \in R^{n}$ that contains the estimated moments. $\sigma n \epsilon R^{n}$ is a noise at the instant $t$ which is assumed Gaussian and $t_{i, j}$ is the instant when the packet $j$ is received at the Time Slot $i$, which is also assumed Gaussian. We suppose that we know perfectly $\sigma^{2}$. The equation can be written in the form:

$$
Y=P W T+\sigma n
$$

As results of the prediction mechanism computation, we will have a vector that contains instants when the node has to turn on its radio interface to probably receive data. These instants must necessarily be adjusted in order that they are in the active part interval of the super-frame $\left[0, S D\left(S D=2^{S O} \mathrm{x}\right.\right.$ aBaseSuperframeDuration)]. where SO means the Super-frame Order, and aBaseSuperframeDuration is a constant equal to $15.36 \mathrm{~ms}$. Eq. (4) allows us to precisely adjust the estimated instants.

$$
Y_{\text {adjasted }}=\left\lfloor\frac{\text { Yestimed }- \text { bcnR } x \text { Time }}{\text { aBaseSuperframeDuration }}\right\rfloor \mid
$$

Where bcnRxTime means the time of the last received beacon, all $Y_{\text {adjasted }}$ values belong to the interval $[0, S D] \epsilon N$, and which exactly correspond to time slot numbers in which the node may receive data.

Thanks to this prediction mechanism the network nodes become in active mode only when they probably have data to receive, Therefore, it is possible for given communication to have a destination which will unreachable for the moment, which can negatively influence on the PDR by increasing the packets lost. To cope with this 


\begin{tabular}{|c|c|c|c|c|}
\hline $\begin{array}{c}\text { Octets: } \\
2\end{array}$ & 1 & 2 & 2 & 2 \\
\hline $\begin{array}{c}\text { Frame } \\
\text { Control }\end{array}$ & $\begin{array}{c}\text { Sequence } \\
\text { Number }\end{array}$ & $\begin{array}{c}\text { ID of } \\
\text { Concerned } \\
\text { Node }\end{array}$ & $\begin{array}{c}\text { Instant } \\
\text { of First } \\
\text { attempt }\end{array}$ & $\begin{array}{c}\text { FCS } \\
\text { (Frame } \\
\text { Chek } \\
\text { Sequence) }\end{array}$ \\
\hline \multicolumn{2}{|c|}{$\begin{array}{c}\text { MHR (MAC } \\
\text { Header) }\end{array}$} & \multicolumn{2}{|c|}{ MAC Payload } & $\begin{array}{c}\text { MFR } \\
\text { (MAC } \\
\text { Footer) }\end{array}$ \\
\hline
\end{tabular}

Figure. 3 Data postponing frame

Table 1. Simulation configuration

\begin{tabular}{|c|c|}
\hline Parameters & Value \\
\hline Topology Dimension & $300 \times 300 \mathrm{~m}^{2}$ \\
Type of topology & Mesh \\
Propagation mode & Two-Ray-Ground \\
Routing protocol & AODV \\
Data Packet Size & $100 \mathrm{~B}$ \\
Radio power in sleep mode & $0.015(\mathrm{~mW})$ \\
Radio power in idle and listen mode & $14.4(\mathrm{~mW})$ \\
Radio power in transmitting mode & $36.0(\mathrm{~mW})$ \\
Temps de Simulation & $1000(\mathrm{~s})$ \\
Nodes Numbers & 100 \\
\hline
\end{tabular}

challenge, AD-MAC protocol has created a new procedure which consists in the first step to postpone the last transmission attempt to the next super-frame, then, send a new packet called data postponing frame (see Fig.3) to the coordinator node of the network. This new packet is intended to let the coordinator node know that the $\mathrm{Y}$ node (destination) was interrogated by the $\mathrm{X}$ node (source) at time $t x$, this information will be delivered to the $\mathrm{Y}$ node (destination) through the next beacon frame, for this purpose, AD-MAC protocol has made a change in the beacon frame by adding two new fields, the first field ( 2 octets) shall contain the destination node identifier, while the second field ( 2 octets) shall contain the instant of the first transmission attempt. And as we mentioned previously, at the beginning of each super-frame the coordinator node broadcasts the beacon frame, this frame will be received by all network nodes, but only the one which have its identifier equal to that inserted in the first new field can read the second field content in order to include it into the prediction mechanism computation.

The data postponing frame shall be constructed as illustrated in Fig.3, and the fields order of this packet shall respect the order of the general MAC frame [19].

The MHR for a data postponing frame shall contain only the frame control field and the sequence number field. In the Frame Control field, the frame type subfield shall contain a value different to these used by the beacon, Data, acknowledgment, and the MAC command. We propose using the value 7 to indicate a data postponing frame. The Sequence Number field shall contain the same value of the data frame sequence number. The MAC payload for a data postponing frame shall contain two fields: the first field contains the concerned node identifier, while the second field contains the instant when the first attempt of transmission occurred. The MFR is composed of a 16 bit FCS.

\section{Performance evaluation}

To validate our numerical analysis, we evaluate the model under different numbers of nodes, different types of traffic, and compare the results of the PDR, End-to-End delay and the sum of the energy consumption obtained by the AD-MAC protocol with those of the IEEE 802.15.4 standard and SMAC protocol using the Network Simulator (NS-2). We have performed simulation based evaluations to validate AD-MAC protocol. Hence, we consider 99 sensor nodes connected to coordinator node and which are randomly deployed in the network. In the simulation, each sender node randomly selects one of its neighbours as the destination. In the first stage of our simulation the sender node generates event-driven traffic load characterized by the ON/OFF exponential traffic. While, in the second simulation step sensor node generates CBR (Constant Bit Rate) traffic. All results are the average of 30 simulations. The simulation parameters are illustrated in Table 1.

\subsection{Simulation results under burst traffics}

In this section, the PDR, energy consumption and average latency are evaluated using the burst traffic. Network performance metrics are examined to analysis the impact of communications load and the impact of the traffic density on AD-MAC protocol behaviour.

\subsubsection{Results of energy consumption}

Extending the overall network lifetime is the most important challenge to resolve in WSNs, though, several protocols have been proposed to improve network lifetime. Using network simulation, It is found that when sender nodes number or ON period of the burst traffic increases the energy consumption increases as well for each of IEEE 802.15.4, SMAC and AD-MAC protocol. In Fig.4 we observe that the AD-MAC protocol greatly outperforms IEEE 802.15.4 standard and SMAC 

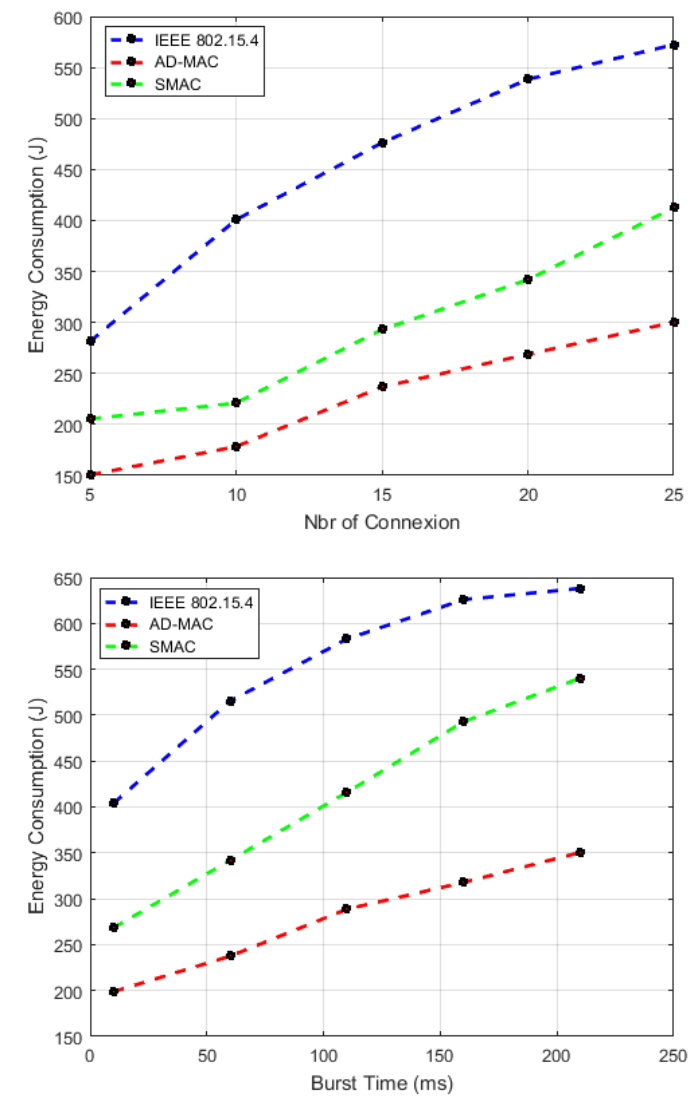

Figure. 4 Energy consumption results
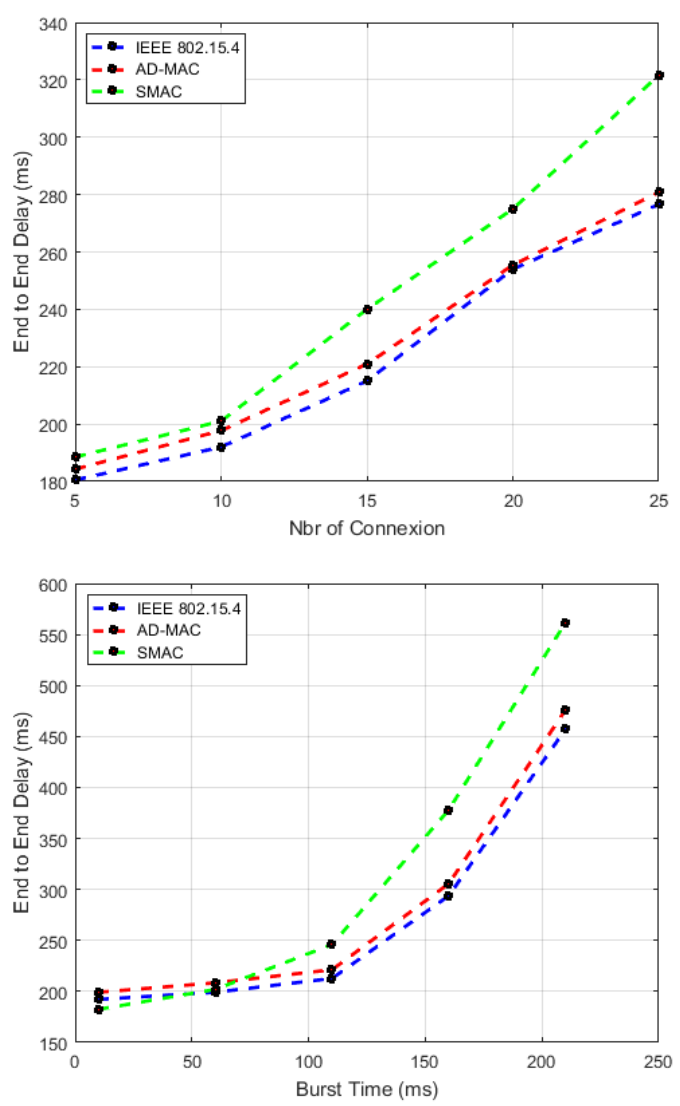

Figure. 5 Latency results protocol in term of minimizing the energy consumption. This is can be explained by the fact that the AD-MAC protocol does waken up the sensor nodes precisely when they have high probability to receive data. Hence, network nodes become active less frequently and considerably reduce their energy consumption due to idle listening, In contrast to the SMAC and IEEE 802.15.4 protocols which are based on a classical duty-cycling to control the wake up / sleep process of the sensor nodes.

\subsubsection{Results of latency}

The time duration between the receive and the send moment of packet depicts the stability and reliability of the WSN network. More the end to end delay is reduced, the algorithm is found to be more stable. Normally, when traffic density or communication load becomes high in network, the delivery end-to-end delay also becomes significant because of the regular packets retransmission. Fig.5 shows that there is a small difference between ADMAC protocol latency and the one of IEEE 802.15.4 standard and SMAC protocol. This can be explained by when a node send data packet to a destination that goes into sleep mode because of its prediction mechanism, therefore, the last transmission attempt of this packet will be postponed to the next superframe which will causes an additional delay.

\subsubsection{Results of PDR}

The PDR is the ratio between the number of received packets and the number of sent packets. For an efficient network, the PDR should be a maximum value. Higher PDR increases the reliability of the network. Fig.6 shows that the ADMAC protocol largely exceeds the IEEE 802.15.4 standard especially in cases of low and medium traffic load. These results are proved by the accuracy of the prediction mechanism, because we estimate exactly when a node must to wake up for receiving its data efficiently. In addition, even if we increase the traffic load in the network, the PDR of AD-MAC protocol remains better than the PDR obtained by IEEE 802.15.4 standard and SMAC protocol. Using AD-MAC protocol, the number of the lost packets (due to the collision or when the destination is unreachable) is reduced thanks to the packet "Data postponing frame", which allows to postpone the last transmission attempt to the next super-frame where the receiver node is surely active. 

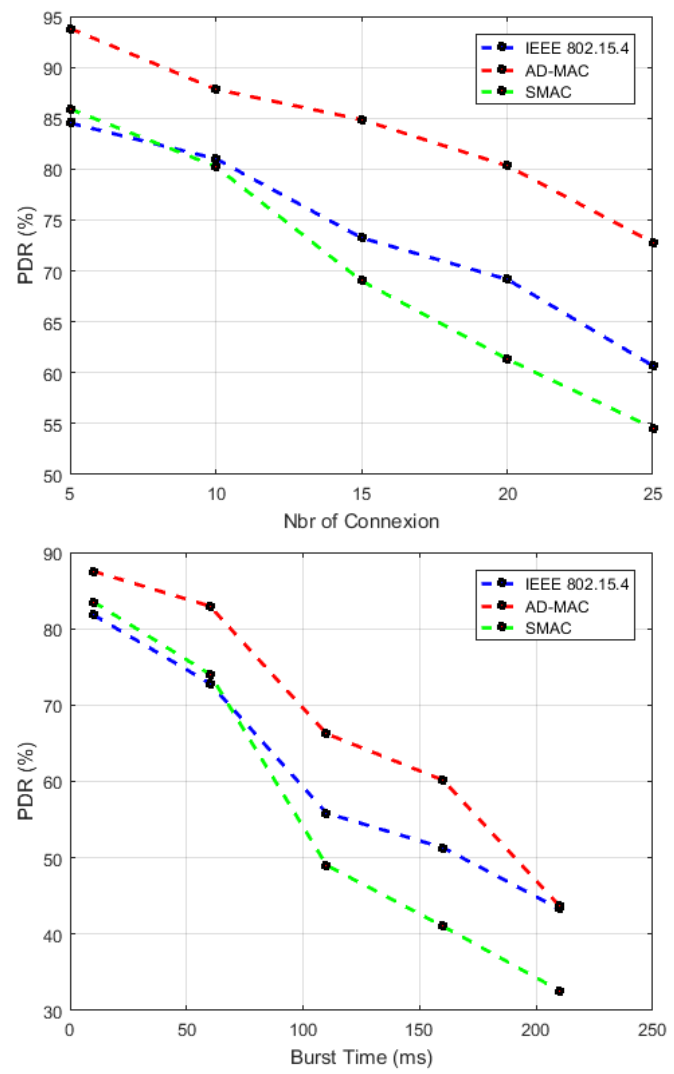

Figure. 6 PDR results

\subsection{Simulation results under CBR traffics}

In this section, AD-MAC protocol behaviour is examined using the CBR traffic. Network performance metrics are evaluated in first step by fixing the communications number at 10, and varying the packets transmission interval from $0.5 \mathrm{~s}$ to $2.5 \mathrm{~s}$. While in second step of this simulation, the packets transmission interval between the events is fixed at $1.5 \mathrm{~s}$. Then, we varied the communications number from 5 to 25 .

\subsubsection{Results of energy consumption}

The results shown in Fig.7 prove the efficiency of the AD-MAC protocol in terms of energy conservation thanks to its prediction process that vastly reduced the idle listening. According to these figures, the AD-MAC protocol perfectly arrives to adapt the traffic load with the duty cycle scheme. It is thanks to the fact of the sensor nodes will activate their radio interface only if they probably have data to transmit or to receive. Otherwise, the radio interface will remain inactive and preserve more energy. Therefore, AD-MAC protocol presents a better solution for efficient energy consumption. This clearly depicts the stability of the proposed protocol in comparison to the IEEE 802.15.4 and SMAC protocols.
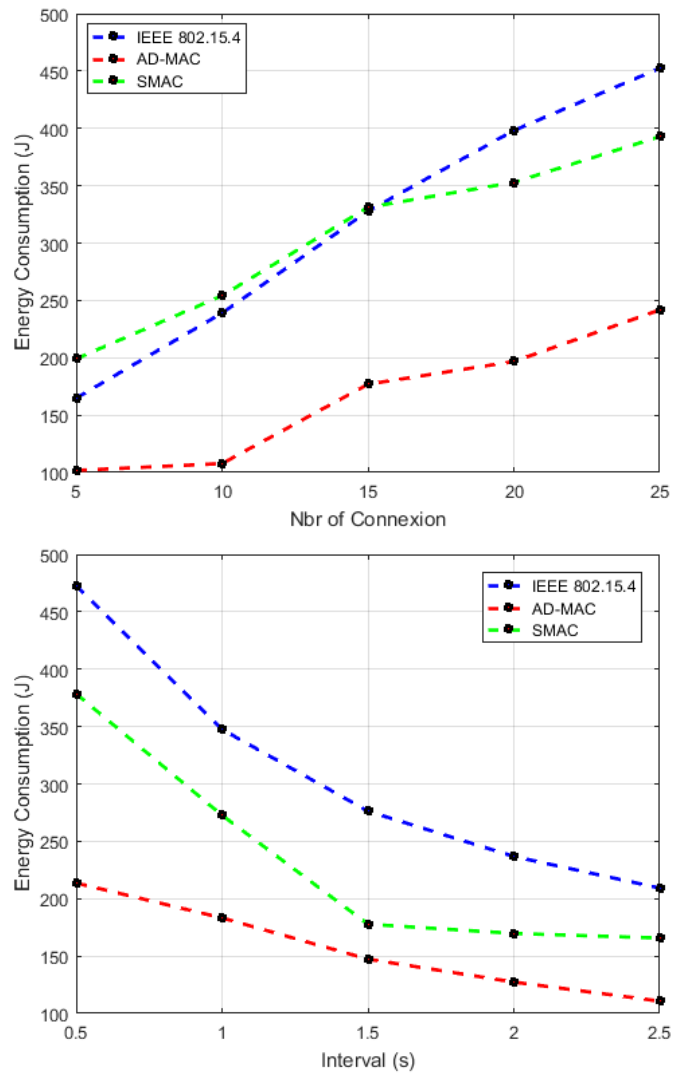

Figure. 7 Energy consumption results

\subsubsection{Results of latency}

In Fig. 8 we can see that there is no difference between delivery end-to-end delay obtained by ADMAC protocol and the one of IEEE 802.15.4 standard even we increase the communication load, which proves that the number of retransmitted packets (postponed packets) is decreased. In these experiments we used a CBR traffic which is characterized by periodic events generation. Therefore, we can say that the prediction process converges to correctly estimate the events generation periods. Consequently, we don't need to frequently postponing the transmission to the next super-frame when we use the CBR traffic, which will reduce the latency.

\subsubsection{Results of PDR}

The depicted results shown in Fig.9 show that there is a significant difference between the both curves, especially when the traffic load in the network is low or medium. AD-MAC protocol improves the PDR by minimizing packet loss due to collision. AD-MAC achieved these results thanks to the packet postponing procedure which allows the sender node to postpone the last retransmission attempt to the next super-frame where the destination node is probably reachable. 

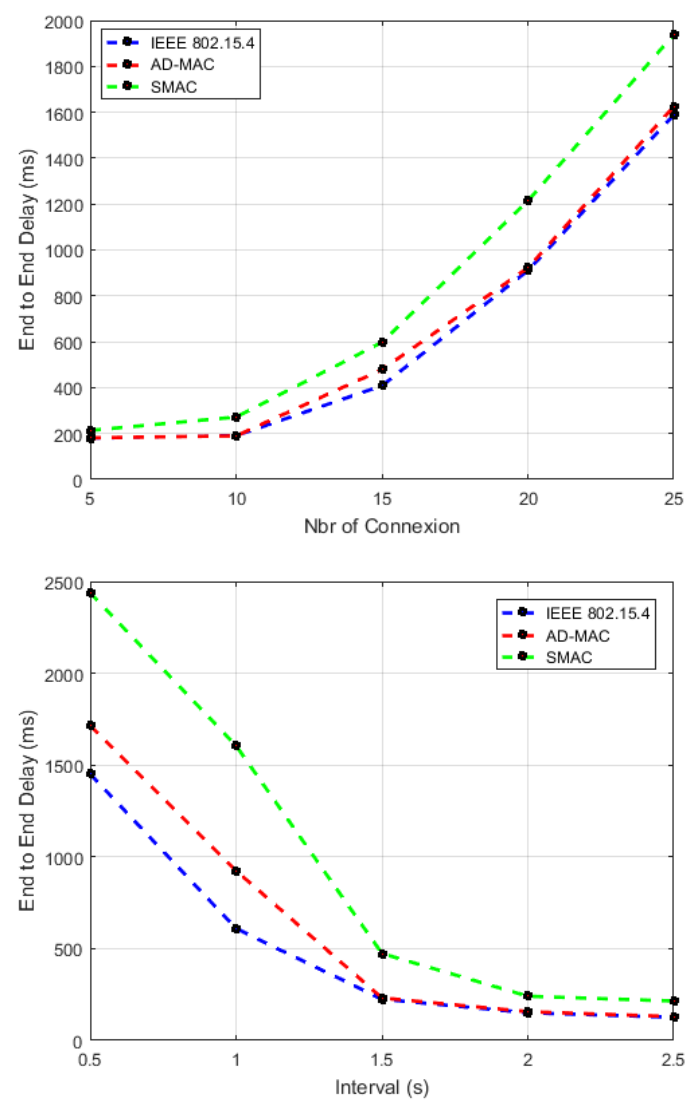

Figure. 8 Latency results
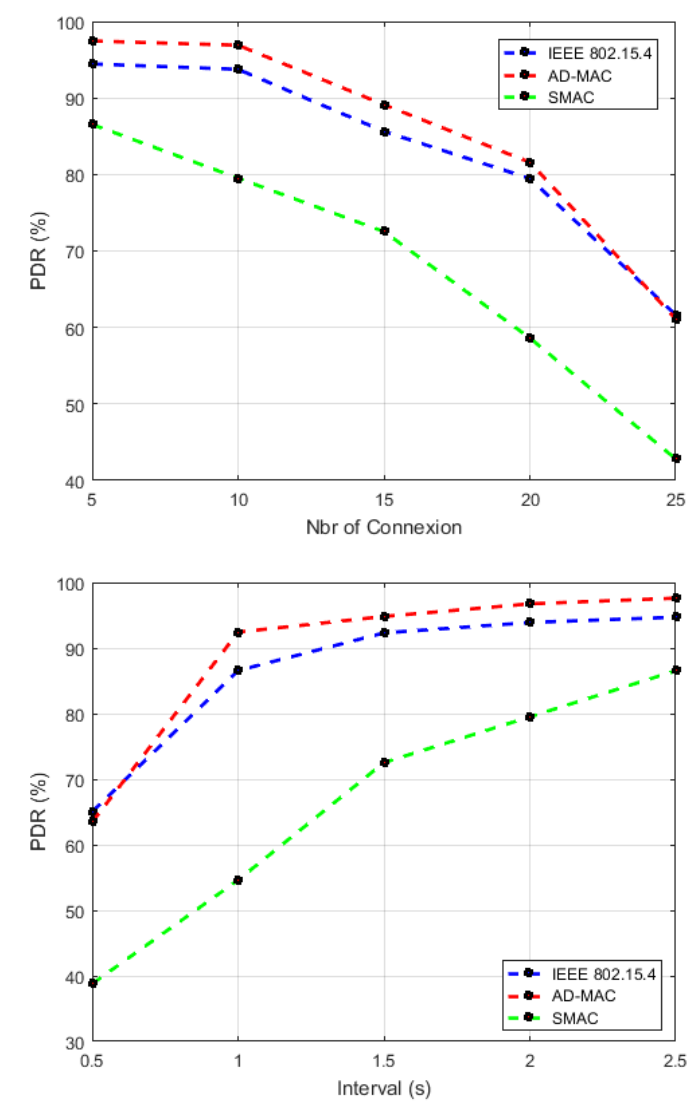

Figure. 9 PDR results

\section{Conclusion}

In this article, we proposed a simple yet effective energy-efficient MAC protocol called AD-MAC for energy minimizing in wireless sensor networks. ADMAC periodically adjusts each node's super-frame structure according to its previous traffic load. And the iteration of this adjustment quickly leads all network nodes to optimize efficiently the energy and improve the PDR without causing a large transmission delay. AD-MAC works in a full distributed manner, no requiring to any central computation, global time synchronization or the knowledge of nodes state, and brings no additional load to network. Simulation results via NS2 simulator validates that AD-MAC has better performance than IEEE 802.15.4 and SMAC protocols. A direction of our future work would be an extension of the AD-MAC protocol for improving the latency to be suitable to real time applications, and we intend adapting the AD-MAC protocol in different application environments..

\section{References}

[1] M. P. Đurišic, Z. Tafa, G. Dimic, and V. Milutinovic, "A survey of military applications of wireless sensor networks", In: Proc. of Embedded Computing, 2012 Mediterranean Conference on IEEE, pp. 196-199, 2012.

[2] J. Ko, C. Lu, M. B. Srivastava, J. A. Stankovic, A. Terzis, and M. Welsh, "Wireless sensor networks for healthcare", In: Proc. of the IEEE, Vol. 98, No 11, pp. 1947-1960, 2010.

[3] D. Puccinelli and M. Haenggi, "Wireless sensor networks: applications and challenges of ubiquitous sensing", IEEE Circuits and Systems Magazine, Vol. 5, No. 3, pp. 19-31, 2005.

[4] M. Stemm and R. H. Katz, "Measuring and Reducing Energy Consumption of Network Interfaces in Hand-Held Devices", IEICE Transactions on Communications, Vol. 80, No. 8, pp. 1125-1131, 1997.

[5] I. Dbibih, O. Zytoune, and D. Aboutajdine, "On/off markov model based energy-delay aware mac protocol for wireless sensor network", Wireless Personal Communications, Vol. 78, No. 2, pp. 1143-1155, 2014.

[6] Y. Zhan, Y. Xia, and M. Anwar, "GTS size adaptation algorithm for IEEE 802.15.4 wireless networks", Ad Hoc Networks, Vol. 37, pp. 486498, 2016.

[7] F. Cuomo, A. Abbagnale, and E. Cipollone, "Cross-layer network formation for energy- 
efficient IEEE 802.15.4/ZigBee Wireless Sensor Networks", Ad Hoc Networks, Vol. 11, No. 2, pp. 672-686, 2013.

[8] K. H. Jung, H. R. Lee, W. S. Lim, and Y. J. Suh, "An adaptive collision resolution scheme for energy efficient communication in IEEE 802.15. 4 networks", Computer Networks, Vol. 58, pp. 39-57, 2014.

[9] M. H. S. Gilani, I. Sarrafi, and M. Abbaspour, "An adaptive CSMA/TDMA hybrid MAC for energy and throughput improvement of wireless sensor networks", Ad Hoc Networks, Vol. 11, No. 4, pp. 1297-1304, 2013.

[10] W. Ye, J. Heidemann, and D. Estrin, "An energy-efficient MAC protocol for wireless sensor networks", In: Proc. of the Twenty-First Annual Joint Conference of the IEEE Computer and Communications Societies, Vol. 3. pp. 1567-1576, 2002.

[11] R. de Paz Alberola and D. Pesch, "Duty cycle learning algorithm (DCLA) for IEEE 802.15. 4 beacon-enabled wireless sensor networks", $A d$ Hoc Networks, Vol. 10, No. 4, pp. 664-679, 2012.

[12] A. K. Subramanian and I. Paramasivam, "PRIN: A Priority-Based Energy Efficient MAC Protocol for Wireless Sensor Networks Varying the Sample Inter-Arrival Time", Wireless Personal Communications, Vol. 92, No. 3, pp. 863-881, 2017.

[13] F.A. Aoudia, M. Gautier, and O. Berder, "OPWUM: Opportunistic MAC protocol leveraging wake-up receivers in WSNs", Journal of Sensors, Vol. 2016, 2016.

[14] J. Oller, I. Demirkol, J. Casademont, J. Paradells, G. U. Gamm, and L. Reindl, "Has time come to switch from duty-cycled MAC protocols to wake-up radio for wireless sensor networks?", IEEE/ACM Transactions on Networking, Vol. 24, No. 2, pp. 674-687, 2016.

[15] Y. H. Zhu, H. Lv, Y. Li, E. Li, and K. Chi, "Energy Conservation Scheme for IEEE 802.15. 4 Based Battery-Free Wireless Sensor Networks. In Networking and Network Applications (NaNA)", International Conference on IEEE, pp. 342-348, 2016.

[16] N. Hiremani and T. G. Basavaraju, "An Efficient Routing Protocol Adopting Enhanced Cluster Formation Technique Accompanied by Fuzzy Logic for Maximizing Lifetime of WSN", International Journal of Intelligent
Engineering and Systems, Vol.9, No. 4, pp. 185-194, 2016.

[17] S. Chandra, K. K. Patil, and S. Talanki, "CrossLayer Interactive Framework for Enhancing Network Lifetime and Communication in WSN", International Journal of Intelligent Engineering and Systems, Vol.10, No. 6, pp. 263-272, 2017.

[18] D. Onthachi and S. Jayabal, "An Optimized QoS-based Multipath Routing Protocol for Wireless Sensor Networks", International Journal of Intelligent Engineering and Systems, Vol. 11, No. 2, pp. 49-56, 2018.

[19] LAN/MAN Standards Committee, "Part 15.4: wireless medium access control (mac) and physical layer (phy) specifications for low-rate wireless personal area networks (lr-wpans)", IEEE Computer Society, 2003. 